A N N A L E S Annales de Bretagne et des Pays de l'Ouest

Anjou. Maine. Poitou-Charente. Touraine

123-1 | 2016

Varia

\title{
Maupertuis et la science de la musique
}

\section{Marco Storni}

\section{OpenEdition}

\section{Journals}

Édition électronique

URL : http://journals.openedition.org/abpo/3211

DOI : $10.4000 / a b p o .3211$

ISBN : 978-2-7535-5040-7

ISSN : 2108-6443

Éditeur

Presses universitaires de Rennes

Édition imprimée

Date de publication : 22 avril 2016

Pagination : 157-178

ISBN : 978-2-7535-5038-4

ISSN : 0399-0826

Référence électronique

Marco Storni, «Maupertuis et la science de la musique », Annales de Bretagne et des Pays de l'Ouest [En ligne], 123-1 | 2016, mis en ligne le 22 avril 2018, consulté le 20 avril 2019. URL : http:// journals.openedition.org/abpo/3211; DOI : 10.4000/abpo.3211 


\title{
Maupertuis et la science de la musique
}

\author{
Marco STORNI ${ }^{1}$ \\ Doctorant, USR 3608 - La République des Savoirs, ENS, Paris - \\ Dipartimento di Filosofia e Comunicazione, Università di Bologna
}

Pourquoi consacrer une étude à la pensée du " savant oublié ${ }^{2}$ " Pierre Louis Moreau de Maupertuis (1698-1759)? Tout d'abord, parce que nous pourrions mettre en exergue l'obscurité dans laquelle Maupertuis est tombé depuis deux siècles, et souligner par la même occasion le rôle central que le savant malouin a joué sur la scène intellectuelle de son époque. Mais alors, pour quelles raisons choisissons-nous d'aborder l'étude de son premier mémoire académique portant sur la forme des instruments de musique, texte qu'il rédigea dans ses jeunes années, et non ses travaux de maturité qui, eux, ont été davantage débattus et influents? D'abord, parce que l'historiographie s'est moins concentrée sur les écrits de jeunesse de Maupertuis, écrits qui, somme toute, témoignent d'une originalité remarquable, laquelle échappe souvent aux commentateurs. Deuxièmement, l'analyse des premiers travaux maupertuisiens sera notamment pour nous l'occasion d'approfondir les étapes principales de sa première formation intellectuelle, aussi bien que les sources possibles de quelques-unes de ses doctrines. En effet, une telle enquête fut constamment négligée, faute de documents ainsi que de témoignages directs ayant un lien avec les premières années de sa carrière. Nous proposerons ainsi, en plus d'une analyse textuelle rigoureuse, une étude de nature socioculturelle sur les milieux érudits que fréquentait Maupertuis en arrivant à Paris depuis la Bretagne, au début des années 1720 . Nous espérons de cette façon apporter un nouvel éclairage sur les débuts de son activité, de même que sur le caractère de ce personnage et les relations qu'il entretenait avec d'autres savants.

1. Je tiens à remercier Martin Jaillet et Jean-Daniel Thumser pour avoir corrigé mon français, ainsi que les rédacteurs des $A B P O$, notamment $\mathrm{M}^{\mathrm{me}}$ Annie Antoine et $\mathrm{M}$. Gauthier Aubert, pour leur patience. Mes remerciements aussi tout particulièrement aux directrices de mes recherches, $\mathrm{M}^{\text {mes }}$ Sophie Roux et Mariafranca Spallanzani, pour toutes leurs corrections et leurs conseils.

2. VALENTIN, Michel, Maupertuis : un savant oublié, Rennes, La Découvrance, 1998. 


\section{"Sur la forme des instruments de musique "}

Les premiers travaux scientifiques de Maupertuis sont généralement considérés comme l'œuvre d'un pur géomètre. Quelques commentateurs ont toutefois remarqué l'importance d'autres centres d'intérêt chez le jeune Maupertuis, notamment dans le domaine de la biologie ${ }^{3}$. Mais il reste à examiner un certain nombre de questions cruciales pour bien comprendre les débuts du parcours intellectuel maupertuisien. Le point de départ le plus naturel est le tout premier travail de Maupertuis, à savoir le mémoire intitulé Sur la forme des instruments de musique, qu'il lut à l'Académie des sciences de Paris le 15 novembre $1724^{4}$ lors d'une séance publique ${ }^{5}$.

Dans le recueil des Mémoires de mathématique et de physique tiré des registres de l'Académie royale des sciences pour l'année 1724, le travail du savant malouin occupe la rubrique Acoustique, laquelle n'est évoquée que rarement dans ce type de publication. Dans la période 1699-1724, il n'existe en fait que peu de travaux portant sur l'acoustique, dont on mentionnera notamment ceux de Louis Carré (1663-1711) et de Philippe de La Hire (1640-1718). Le point fondamental de leurs recherches, comme Fontenelle le rappelle dans l'Histoire de l'Académie Royale des Sciences de l'année 1716 , était de montrer " que le Son n'est pas produit, comme on le pourrait croire, par les vibrations totales du corps sonore, mais par les vibrations particulières de toutes ses petites parties ${ }^{6}$ ". À première vue, l'étude de Maupertuis semble en fait assez proche de ce type de travaux : en la schématisant, on pourrait réduire la base théorique du mémoire maupertuisien à l'idée - effectivement très semblable à celle de Carré et de La Hire - selon laquelle le son issu des instruments de musique est toujours le produit d'un mouvement global de toutes leurs "petites parties".

Or, ce qui nous frappe le plus, c'est que les spécialistes de la pensée de Maupertuis, ainsi que ses biographes, mentionnent l'ouvrage de 1724 sans s'interroger sur son intérêt historique ni sur sa portée théorique. Pour n'en nommer que quelques-uns, La Beaumelle dans sa Vie de Maupertuis (1856) n'évoque que le titre du travail ${ }^{7}$, et Pierre Brunet dans ses ouvrages classiques de 1929, Maupertuis. Étude biographique et L'œuvre et sa place dans la pensée scientifique et philosophique du XVIII siècle, ne va pas beaucoup plus loin ${ }^{8}$. Plus récemment, Émile Callot, dans Maupertuis : le savant et le

3. Ostoya, Paul, "Maupertuis et la biologie ", Revue d'histoire des sciences et de leurs applications, VII, 1954 , p. $60-78$.

4. Mémoires de l'Académie Royale des Sciences, 1724, p. 215-226.

5. Voir le compte rendu détaillé qu'en donne le Mercure de France dans le numéro de novembre 1724, p. 2425-2431.

6. Histoire de l'Académie Royale des Sciences, 1716, p. 66.

7. La Beaumelle, Laurent Angliviel de, Vie de Maupertuis, ouvrage posthume suivi de Lettres inédites de Frédéric le Grand et de Maupertuis, Paris, Ledoyen et Ch. Meyrueis, 1856, p. 15.

8. BRUnet, Pierre, Maupertuis. Étude biographique, Paris, Blanchard, 1929, p. 13. BRUNET, Pierre, Maupertuis. L'œuvre et sa place dans la pensée scientifique et philosophique du 
philosophe (1964) semble avoir oublié l'existence du petit travail juvénile ${ }^{9}$, et Giorgio Tonelli, dans son étude très érudite La pensée philosophique de Maupertuis : son milieu, ses sources (1987), a estimé que ce mémoire n'était pas digne d'être mentionné ${ }^{10}$. Le désintérêt manifesté par les commentateurs envers ce texte est d'autant plus étonnant si l'on considère les difficultés évidentes qu'il pose d'un point de vue à la fois historique et théorique.

La première tient à la place éminente qu'y occupe la réflexion épistémologique. Dans plusieurs passages du texte, en effet, la discussion savante ayant pour objet la forme des instruments de musique laisse la place à une réflexion de nature philosophique, intéressée aux implications méta-théoriques de la discussion plus qu'à la détermination détachée des avantages et des inconvénients d'une hypothèse scientifique quelconque. Vers la moitié du texte, Maupertuis imagine ainsi une objection à son système et il en donne tout de suite la réfutation. Extrêmement intéressante est la conclusion plus générale que le savant croit tirer de ce petit exercice dialectique :

"Les expériences, conformes en tout à un système physique, ne le confirment peut-être pas tant que celles qui paraissent d'abord lui être contraires, et qui, mieux examinées, se soumettent aux lois du système, c'est une espèce de dédommagement que l'expérience rebelle doit au Physicien pour l'alarme qu'elle lui avait causée ${ }^{11}$."

De façon similaire, à la fin du mémoire, une importance particulière est accordée à quelques considérations de nature méta-scientifique. "L'explication de tous ces phénomènes, écrit Maupertuis, [...] n'est cependant toujours qu'une hypothèse physique, et par conséquent assujettie à un certain degré d'incertitude qui est essentiellement attachée à ces sortes des matières ${ }^{12}$ ". L'incursion dans l'épistémologie hasardée ici par Maupertuis n'est pourtant pas aussi improvisée qu'on pourrait le croire à première vue; on peut en fait y entrevoir quelques éléments qui seront tout à fait caractéristiques de l'épistémologie du Maupertuis de la maturité ${ }^{13}$. On lit par exemple dans l'ouvrage de 1724 :

XVIII ${ }^{e}$ siècle, Paris, Blanchard, 1929, p. 280-288; dans ces pages, Brunet ne fait rien de plus que résumer l'ouvrage de Maupertuis.

9. CALlot, Émile, Maupertuis : le savant et le philosophe. Présentation et extraits, Paris, Rivière, 1964. L'ouvrage de Maupertuis le plus ancien mentionné par Callot est le Discours sur les différentes figures des astres, paru en 1732.

10. Tonelli, Giorgio, La pensée philosophique de Maupertuis. Son milieu et ses sources, édition posthume par Claudio Cesa, Hildesheim, Georg Olms, 1987. Le mémoire Sur la forme des instruments de musique n'est même pas mentionné dans la notice biographique préliminaire (ibid., p. 6).

11. Mémoires de l'Académie Royale des Sciences, 1724, p. 221.

12. Ibidem, p. 226.

13. Par " épistémologie du Maupertuis de la maturité ", on entend la théorie " ultraphénoméniste " de la connaissance faisant son entrée dans l'œuvre du savant à partir des années 1740, notamment avec la publication des Réflexions philosophiques sur l'origine des langues et la signification des mots (cf. TONELLI, Giorgio, La pensée philosophique..., op. cit., p. 8). 
"Il faut s'y [en physique] contenter des conjectures; mais il y a des conjectures de différents degrés, toutes plus ou moins éloignées de la certitude; et il y en a qui en approchent si fort, qu'on les prendrait pour elle, ou du moins on pourrait soupçonner celui qui les propose, de s'y être trompé lui-même ${ }^{14}$."

Or, la tendance des commentateurs consiste généralement à placer l'émergence des intérêts philosophiques chez Maupertuis après son installation à Berlin comme président de l'Académie royale des sciences de Prusse en $1746^{15}$. Cependant, la présence de réflexions de nature épistémologique dans cet ouvrage de jeunesse oblige à reformuler, au moins en partie, cette thèse historiographique. Une activité de révision en ce sens a été déjà entreprise par David Beeson, qui a suggéré, avec des arguments fort convaincants, d'antidater les Réflexions philosophiques sur l'origine des langues et la signification des mots de 1748 à 1740, en démontrant ainsi que cet ouvrage, de loin le plus philosophique de Maupertuis, est véritablement un ouvrage de jeunesse et non pas tardif ${ }^{16}$. Malheureusement, la voie inaugurée par Beeson n'a pas été suivie jusqu'au bout par les recherches ultérieures.

Une deuxième difficulté est représentée par l'attention que Maupertuis manifeste à plusieurs reprises envers l'histoire des sciences - en l'espèce, l'histoire de la théorie musicale ou de l'art de construire des instruments de musique. Pour enrichir son discours, le savant y ajoute en fait des conjectures historiques (on pourrait dire aussi " philosophico-historiques "). Plus qu'être des savants médiocres, écrit-il, les Anciens étaient aussi de piètres artisans ${ }^{17}$. Par conséquent, de l'Antiquité jusqu'au XVII ${ }^{\mathrm{e}}$ siècle les progrès dans l'art de construire les instruments de musique n'ont pas été le résultat de calculs précis ou de démonstrations mathématiques, mais plutôt d'une recherche effectuée par tâtonnements. Maupertuis, toutefois, fait bien attention à ne pas déprécier de façon simpliste le chemin historique, toujours précaire et difficile, de l'art ou de la technique de fabriquer les instruments. "La voie du tâtonnement ", dit-il, " est souvent bien longue, mais elle est presque toujours la plus sûre. On va voir que le temps a donné aux Instruments la forme que la Physique leur prescrivait ${ }^{18}$ ". Il est vraiment frappant de retrouver des considérations pareilles dans une communication académique, surtout lorsque l'on considère qu'il s'agit de l'œuvre d'un néophyte. Il nous semble donc logique de nous demander pourquoi Maupertuis a ressenti le besoin d'insérer dans son texte de telles remarques.

14. Mémoires de l'Académie Royale des Sciences, 1724, p. 226.

15. Cf. Tonelli, Giorgio, La pensée philosophique..., op. cit., p. 3-5; Terrall, Mary, The Man Who Flattened the Earth. Maupertuis and the Sciences in the Enlightenment, Chicago/ London, The University of Chicago Press, 2002, p. 265-286.

16. BEEson, David, "Maupertuis at the Crossroads: Dating the Réflexions philosophiques ", Studies on Voltaire and the Eighteenth Century, 249, 1987, p. 241-250.

17. Mémoires de l'Académie Royale des Sciences, 1724, p. 216.

18. Ibidem, p. 217. 
Enfin, il demeure un dernier aspect problématique concernant la teneur technique du travail de Maupertuis. Comme on le sait, l'Académie des sciences a toujours placé l'utilité parmi ses objectifs principaux, entre autres pour des raisons d'ordre politique. Comme le secrétaire perpétuel de l'Académie entre 1699 et 1737, Fontenelle, affirme dans son Discours préliminaire sur l'utilité des Mathématiques et de la Physique ${ }^{19}$, l'utilité des pratiques savantes de l'Académie " est invisible à la plupart du monde ${ }^{20}$ ", car elle ne se manifeste que d'une façon médiate. L'art de la navigation, selon l'exemple donné par Fontenelle lui-même, ne peut pas se développer sans un progrès préalable de l'astronomie, ce qui n'implique pas, bien entendu, que les astronomes interagissent en personne avec les navigateurs. C'est justement pour sa relation avec la pratique de la navigation que Fontenelle se sent autorisé à définir l'astronomie comme une "science utile ". Le mémoire de Maupertuis se trouve par contre traversé par une notion d'utilité tout à fait différente de celle que thématise Fontenelle. Si, chez ce dernier, science et art sont deux domaines nettement séparés qui s'influencent indirectement, chez Maupertuis en revanche, science et art dialoguent directement. Dans le mémoire Sur la forme des instruments de musique, on ne trouve pas l'idée d'un développement autonome des sciences auquel les arts devraient ensuite s'adapter (ce qui ferait justement des sciences des savoirs utiles), mais plutôt celle d'un travail de soutien que le savant se charge d'effectuer au profit des artisans, dans le but d'en perfectionner les techniques et les produits finaux :

« Dans la construction des Instruments, il semble qu'on dût tâcher de faire en sorte qu'il ne se trouvât dans les tables et les fonds que des fibres à l'unisson de chaque ton. Un instrument ainsi construit aurait sans doute l'avantage sur les autres, qu'outre qu'il serait plus sonore, le son en serait encore plus net [...]. Si l'on voulait construire des Instruments dans cette vue, il faudrait que les fibres, au lieu qu'elles diminuent insensiblement, diminuassent par sauts, et suivant les longueurs des cordes auxquelles elles se rapporteraient, ce qui donnerait une figure toute différente aux tables, elles seraient terminées par des lignes droites qui feraient des angles droits ${ }^{21}$. "

Il est alors naturel de se demander comment et pourquoi Maupertuis a ainsi structuré son travail, en contrevenant manifestement à la norme habituelle (quasiment une "loi non écrite ") de l'Académie des sciences.

Comment allons-nous procéder par rapport à ces trois problèmes? D'abord, il semble tout à fait raisonnable de supposer qu'un jeune savant à ses débuts a été au moins partiellement guidé par les auteurs et les méthodes qu'il a étudiés lors de sa première formation qui, en 1724, était bien loin d'être achevée. Ainsi, il semble plausible que, si, dans son pre-

19. Publié en guise d'introduction à son Histoire du renouvellement de l'Académie Royale des Sciences de 1719.

20. FonTENelle, Bernard Le Bovier de, Histoire du renouvellement de l'Académie Royale des Sciences en 1699 et Les éloges historiques de tous les académiciens morts depuis ce renouvellement, t. 1, Paris, Brunet, 1719, p. 4.

21. Mémoires de l'Académie Royale des Sciences, 1724, p. 223-224. 
mier mémoire, Maupertuis choisit d'adopter une démarche philosophique, d'ajouter à son discours des remarques philosophico-historiques et finalement d'interpréter d'une façon assez originale le rapport entre science et technique, il avait à l'esprit certains auteurs. Sur ce chemin, toutefois, un obstacle se présente immédiatement. Dans le texte de 1724, on ne trouve pas de référence explicite ni à d'autres auteurs ni à d'autres ouvrages ou mémoires; pire encore, on n'a aucun témoignage direct concernant les relations personnelles du jeune Maupertuis (correspondances, journaux intimes, etc.) : il n'y a donc aucune possibilité de donner des preuves conclusives certifiant ses relations ou ses dettes intellectuelles pour les travaux de ces années.

Comment sortir de l'impasse? Une fois dit qu'il n'y a pas de réponse évidente à la question, nous avons choisi de conduire l'analyse de la manière suivante. Nous allons reconstituer la scène intellectuelle sur laquelle Maupertuis s'est trouvé depuis son arrivée à Paris en 1714, en mettant l'accent sur ses relations avec des auteurs ou des mouvements de pensée selon ce qui est suggéré par les récits biographiques, les éloges ou d'autres sources indirectes. Bien évidemment, cette opération ne nous livrera rien d'absolument certain. Nous ne prétendons évidemment pas que les auteurs et les mouvements intellectuels que nous allons passer en revue aient effectivement joué le rôle des sources pour le mémoire Sur la forme des instruments de musique, ou plus généralement pour la pensée de Maupertuis dans son évolution diachronique : si cette hypothèse est très attachante, insistons sur le fait que nous n'avons aucune preuve attestant d'une influence directe. Dans le pire des cas, toutefois, nous aurons esquissé un cadre historico-philosophique à l'intérieur duquel le savant malouin a débuté sa carrière : une préhistoire de l'activité savante de Maupertuis qui, à ce jour, reste encore largement inexplorée.

\section{Maupertuis à Paris}

Octobre 1714 : le jeune Maupertuis, fraîchement débarqué à Paris, fait son entrée au collège de la Marche où il commence ses études sous la tutelle de Gilles Le Blond ${ }^{22}$. En ce temps là, l'une des doctrines les plus répandues chez les professeurs des collèges est sans doute celle de Descartes. Comme La Beaumelle le rappelle, non sans ajouter une touche de mystification, " le respect superstitieux pour le système de Descartes [...], dans les universités, avait déjà remplacé le culte d'Aristote ${ }^{23}$ ". Toutefois, si l'on fait un panorama de l'enseignement supérieur en France à cette époque, on perçoit effectivement dans de nombreux établissements un certain penchant

22. Gilles Le Blond : professeur de philosophie au collège de La Marche à partir de 1691 à 1705 (cf. Ressources numériques en histoire de l'éducation, Répertoire des professeurs et principaux de la faculté des arts de Paris aux XVII ${ }^{\mathrm{e}}$ et XVIII ${ }^{\mathrm{e}}$ siècles : [http://rhe.ish-lyon. cnrs.fr/?q=pfap-record/5376] site consulté le 16 février 2016)

23. La BeAumelle, Laurent Angliviel de, Vie de Maupertuis..., op. cit., p. 7. 
pour la philosophie cartésienne, ce qui se ressent dans quelques manuels rédigés dans ces années ${ }^{24}$; mais c'est surtout à partir de certaines polémiques soulevées, par exemple par les jansénistes, contre les enseignants des collèges, qui les blâmaient pour avoir " trop d'attachement aux idées nouvelles et surtout à la doctrine de Descartes, pas assez de respect des Anciens et de l'autorité, un penchant périlleux pour les curiosités de la physique, des écarts en théologie ${ }^{25}$ " que l'attrait pour le cartésianisme est perceptible. Dans ce contexte, Maupertuis a effectivement étudié la pensée de Descartes (à vrai dire, quelques-uns de ses ouvrages) dont les arguments ne lui ont guère semblé convaincants. Le jeune savant a alors été poussé à faire de nouvelles lectures philosophiques : La Beaumelle cite notamment les travaux de Malebranche et de Locke ${ }^{26}$. En parallèle, Maupertuis se livrait à l'étude des mathématiques sous la direction de Nicolas Guisnée (†1718), membre de l'Académie royale des sciences. Ce dernier était l'auteur du célèbre traité Application de l'algèbre à la géométrie $(1705)^{27}$, dont le titre - dans la bouche de Carl Boyer - "était adopté tout au long du XVIII ${ }^{\mathrm{e}}$ siècle comme nom d'usage pour ce que Jean Bernoulli avait appelé "géométrie cartésienne" 28 ". Bref, pendant son premier séjour parisien (1714-1716), Maupertuis reçut une éducation de style cartésien qu'il a immédiatement répudiée, comme le fait bien remarquer le physiologiste allemand Emil du

24. On peut se référer, par exemple, au manuel d'Antoine Le Grand, Institutio philosophice, secundum principia Renati Descartes : nova methodo adornata, et explicata. In usum juventutis academicae, Nuremberg, Ziegeri, 1683, et à celui-ci de Guillaume Dagoumer, Philosophia ad usum scholae accomodata, 3 vol., Parisiis, Girin, 1701-1703.

25. JouRDaIN, Charles, Histoire de l'Université de Paris au XVII et au XVIII siècles, t. II, Hachette, Paris, 1862, p. 172, cité par CostaBel, Pierre, L'enseignement classique au XVIII siècle. Collèges et universités, Paris, Hermann, 1986, p. 146. Le même point est remarqué par BrockLISs, L. W. B., French Higher Education in the Seventeenth and Eighteenth Centuries : A Cultural History, Oxford, Clarendon Press, 1987, p. 350-351 : «L'Université de Paris avait été conquise par les mécanistes dans les premières années 1700 et deux décennies après il n'y avait presque des collèges de plein exercice ou de couvent régulier n'ayant succombé à son tour. "

26. Maupertuis ne lisait pas l'anglais : cf. sa lettre à Le Blanc de 28 février 1756 : «Une grande obligation que je vous ai c'est de m'avoir procuré une espèce de connaissance avec M. Hume [...]; je me suis fait traduire ici ses Essais philosophiques, et je les ai lus bien des fois " (Arch. dép. d'Ille-et-Vilaine, Saint-Malo, 1124. f $^{\circ} 117$ v $^{\circ}$ ). Cf. aussi Charles, Sébastien, Berkeley au siècle des Lumières, Paris, Vrin, 2003, p. 185 : " Maupertuis, qui ne lisait pas l'anglais, a pris connaissance des thèses de Berkeley à travers sa lecture de la seconde traduction des Dialogues, celle de 1750. " Il semble donc vraisemblable que Maupertuis ait étudié l'Essai philosophique concernant l'entendement humain dans la version française de 1700. En outre, dans son voyage en Angleterre (1728), Maupertuis a sans doute rencontré Pierre Coste, traducteur de l'Essai en français (les deux fréquentaient en fait la Rainbow Coffee House); peut-être sa connaissance du chef-d'œuvre lockien doit-elle être datée après cet événement. En tout cas, il n'y a pas de traces visibles de l'influence de Locke dans les travaux de Maupertuis avant les années 1730.

27. GuISNÉE, Nicolas, Application de l'Algèbre à la Géométrie, ou Méthode de démontrer par l'Algèbre des Théorèmes de la Géométrie, et d'en résoudre et construire tous les Problèmes, Boudot et Paris, Quillau, 1705.

28. BOYER, Carl Benjamin, History of Analytic Geometry, Princeton, The Scholar's Bookshelf, 1988, p. 149. 
Bois-Reymond dans son célèbre discours Maupertuis : Rede zur Feier des Geburtstages Friedrich's Ii. und des Geburtstages S. M. d. Kaisers (1892) :

"Lorsqu'il eut seize ans, il vint à Paris dans un collège, où pendant deux ans sa soif de connaissance fut apaisée par la philosophie cartésienne. Déjà ici se réveilla en lui de la méfiance envers un Philosophe qui avait commencé par de omnibus dubitandum et avait fini en spéculant de manière extravagante $^{29}$."

Le rapport de Maupertuis avec Descartes est en effet assez problématique : la critique radicale du système cartésien s'accompagne parfois d'une certaine reprise de ses modèles d'argumentation, comme dans la théorie quasi mécaniste proposée dans sa Vénus physique afin d'expliquer le phénomène de la génération, mais surtout dans l'inspiration lato sensu cartésienne, bien visible dans ses premiers travaux mathématiques ${ }^{30}$.

En 1716, le premier séjour parisien de Maupertuis se conclut et celui-ci revient alors à la maison paternelle. Son retrait à Saint-Malo, néanmoins, est de courte durée : dès l'année suivante, il revient dans la capitale. Cette seconde venue à Paris marque le début de l'apprentissage mondain de Maupertuis, lorsque, comme le raconte La Beaumelle, " en mêlant toujours les arts agréables aux arts utiles, il se perfectionna dans ceux de la danse et de la musique ${ }^{31}$ ". Cependant, il devient vite évident que son penchant pour la musique ne pouvait s'arrêter au savoir superficiel qui convient à l'honnête homme : Maupertuis approfondit les principes de la composition musicale avec Nicolas Bernier, célèbre compositeur et claveciniste, " maître de musique de la Sainte-Chapelle, et ensuite de la chapelle du Roi. [...] Ce maître fut, dit-on, le plus grand contrapuntiste qui eût jamais existé en France. L'école qu'il a fondée en ce pays était regardée comme la meilleure ${ }^{32}$ ». Bernier, en théoricien de la musique, introduisit Maupertuis aux concepts et aux débats les plus importants du siècle précédent : le jeune auteur du mémoire Sur la forme des instruments de musique révèle une certaine connaissance des progrès de la science de la musique jusqu'à son époque, et semble aussi avoir médité plusieurs travaux de théorisation de la musique (en l'espèce, Maupertuis mentionne dans son travail le Traité du bruit de Claude Perrault, publié pour la première fois en $1680^{33}$ ).

29. Du Bors-Reymond, Emil, Reden, t. 2, Leipzig, Verlag von Veit \& Comp., 1912, p. 434.

30. Cf. PASSERon, Irène, " Maupertuis, passeur d'intelligibilité. De la cycloïde à l'ellipsoïde aplati en passant par le "newtonianisme" : années parisiennes ", dans HEcht, Hartmut (Hsrg.), Pierre Louis Moreau de Maupertuis. Eine Bilanz nach 300 Jahren, Berlin, Spitz Verlag - Baden-Baden, Nomos Verlagsgesellschaft, 1999, p. 27 : " On voit donc que pour s'en déclarer newtonien, Maupertuis n'en appartient pas moins à une tradition d'écriture mathématique que l'on peut bien appeler "cartésienne". "

31. La Beaumelle, Laurent Angliviel de, Vie de Maupertuis..., op. cit., p. 9.

32. Choron, Alexandre Étienne, FAyolle, François Joseph Marie, Dictionnaire historique des musiciens, artistes et amateurs morts ou vivans, t. 1, Paris, Valade-Lenormant, 1810, p. 70-71.

33. Puis réédité dans les Euvres diverses de physique et de méchanique de Claude et Pierre Perrault, v. 1, t. 2, Leide, Vander, 1721, p. 161-293. Pour des notices biographiques générales sur Claude Perrault voir CHAUDON, Louis Maïeul, Dictionnaire historique, critique 
En outre, Maupertuis apprit à jouer de plusieurs instruments, notamment de la guitare ${ }^{34}$.

Entre 1718 et 1721, Maupertuis se livra à la carrière militaire, en rejoignant d'abord la compagnie des mousquetaires gris, puis un régiment de cavalerie à Lille, sans abandonner ses études pour autant. «En 1718 M. de Maupertuis entra dans les mousquetaires gris ", écrit Formey dans son Éloge (1760), « mais il y porta l'amour de l'étude, et surtout le goût de la géométrie $^{35}$ ". Cependant, son métier de soldat n'allait pas durer longtemps : à la fin de l'année 1721, le savant malouin se rendit finalement, et de façon permanente, à Paris, car il " ne pouvait pas durer longtemps dans l'oisiveté de l'état précédent d'officier militaire en temps de paix, et bientôt il en prit congé ${ }^{36}$ ". Ce moment marque l'entrée officielle de Maupertuis dans la vie intellectuelle parisienne, à mi-chemin entre les cafés littéraires et les bancs de l'Académie. Parmi ses premiers amis, Tressan et Formey mentionnent dans leurs Éloges "Varignon, Saurin, Nicole, Terrasson ${ }^{37}$ ", ceux qui - comme Beeson l'a fait remarquer dans son étude sur Maupertuis (1992) - " ont donné à Maupertuis l'enseignement supérieur en mathématiques et en sciences ${ }^{38}$ ". À noter que les savants nommés étaient tous quatre des membres statutaires de l'Académie royale des sciences : Varignon, élu pensionnaire géomètre le 28 janvier 1699; Terrasson, associé géomètre le 4 décembre 1719; Nicole, adjoint mécanicien le 17 mai 1718; Saurin, pensionnaire géomètre le 13 mai $1707^{39}$. Cependant, ils étaient aussi des visiteurs réguliers des cafés, notamment les cafés Procope et Gradot. Comme le remarque Terrall, Maupertuis «trouva une place agréable dans le cercle autour du critique et poète Antoine Houdar de La Motte, qui était une personnalité incontournable du Gradot ${ }^{40}$ ". D'autres grands noms émergent dans les récits biographiques : nous nous référerons une fois de plus à La Beaumelle, en soulignant que les informations présentées ici par le biographe s'appuient sur des renseignements qui lui étaient fournis par La Condamine (qui en avait une expérience directe ${ }^{41}$ ), et qu'il s'agit donc de notices relativement fiables.

et bibliographique, contenant les vies d'hommes illustres, célèbres ou fameux de tous les pays et de tous les siècles, t. 21, Paris, Menard-Desenne, 1822, p. 384-385.

34. Terrall, Mary, The Man Who Flattened the Earth..., op. cit., p. 33. Voir aussi l'exposé plus détaillé donné par La Beaumelle (Vie de Maupertuis..., op. cit., p. 9-10) : "Il parvint en peu de temps à jouer de divers instruments, en maître. La précision et la délicatesse de son jeu suppléaient à ce degré supérieur d'exécution qu'un longue habitude peut seule donner."

35. Formey, Samuel, Éloge de Monsieur de Maupertuis, Berlin, 1761, p. 15.

36. Du Bois-Reymond, Emil, Reden..., op. cit., p. 434.

37. Formey, Samuel, Éloge ..., op. cit., p. 17; Tressan, Éloge de M. Moreau de Maupertuis..., op. cit., p. 7.

38. BeESON, David, Maupertuis : An Intellectual Biography..., op. cit., p. 62.

39. Ces renseignements sont disponibles à l'adresse internet [www.academie-sciences. fr/acade mie/membre.htm].

40. TERrall, Mary, The Man Who Flattened the Earth..., op. cit., p. 23.

41. SAFIER, Neil, Measuring the New World : Enlightenment Science and South America, Chicago-London, The University of Chicago Press, 2008, p. 27. 
"Le célèbre la Motte-Houdard de l'Académie française, aveugle et infirme dès l'âge de trente ans [...] était insensiblement devenu le dictateur perpétuel d'une république [le cercle d'intellectuels en se réunissant chez Procope] dont les principaux citoyens étaient les La Faye, les Saurin, les Terrasson, les Fréret, les Melon, les Nicole, les Marivaux, les de Pons et d'autres gens de mérite qui vivent encore et dont plusieurs se sont répandus dans les différentes académies ${ }^{42}$."

Il sera utile de nous attarder quelques instants sur les personnages mentionnés dans cette liste, dont les noms sont répétés constamment par tous les biographes de Maupertuis. Bien que, comme nous l'avons dit, l'on ne dispose d'aucun témoignage direct au sujet des relations personnelles du savant à cette époque-là, il semble néanmoins vraisemblable que Maupertuis a eu des contacts avec ces gens et que leur pensée a joué un rôle certain dans sa formation intellectuelle. Une fois de plus, on laissera au lecteur le soin de décider si la discussion suivante ne constitue rien de plus qu'un cadre historico-philosophique au sein duquel Maupertuis a commencé son parcours de recherche, ou bien s'il y a quelque lien plus direct entre les auteurs et les ouvrages que nous allons analyser ci-dessous, et l'approche qu'on retrouve dans le mémoire Sur la forme des instruments de musique. À partir des discussions que nous nous apprêtons à développer, peut-être certaines des difficultés signalées plus haut commenceront-elles à paraître beaucoup moins surprenantes et inexplicables.

\section{Esprits forts}

Nous allons tout d'abord concentrer l'attention sur Fréret. Celui-ci était un membre prééminent de l'Académie des inscriptions, "pensionnaire et secrétaire perpétuel de l'académie des belles-lettres, associé-libre de celle de peinture, membre de celles de Bordeaux et de Cortone ${ }^{43}$ ". En outre, d'après ce qu'en dit Formey, il « fut un des premiers qui s'aperçut de toute l'étendue du mérite, et de toute la force des talents ${ }^{44}$ " de Maupertuis. Quant aux idées philosophiques de Fréret, Jonathan Israel, dans son étude Enlightenment Contested (2006), remarque à plusieurs reprises ses tendances matérialistes et athées : "Ce groupement déterministe, antiscripturaire et matérialiste incluait Fréret, Boindin, Mirabaud, Lévesque de Burigny, Vauvenargues, Du Marsais, La Mettrie, et bientôt Diderot et d'Alembert aussi ${ }^{45}$ ". Ou bien encore :

"Ces intellectuels des Lumières radicales, tous rebelles, qui écrivaient en français étaient Bayle (sauf dans sa politique), Fontenelle, Boulainvilliers, Tyssot de Patot, Lahontan, Fréret, Meslier, Du Marsais, Lévesque de Burigny,

42. La BeAumelle, Laurent Angliviel de, Vie de Maupertuis..., op. cit., p. 11-12.

43. BougainVILle, Louis Antoine de, "Éloge historique de Nicolas Fréret ", dans FréRET, Nicolas, Euvres, t. 1, Paris, Servière et Bastien, 1792, p. I.

44. Formey, Samuel, Éloge de Monsieur de Maupertuis..., op. cit., p. 16-17.

45. IsRael, Jonathan I., Enlightenment Contested. Philosophy, Modernity, and the Emancipation of Man 1670-1752, Oxford, Oxford University Press, 2006, p. 356. 
Boureau-Deslandes, de Maillet, Mirabaud, d'Argens, Boindin, Rousset de Missy, Jean-Frédéric Bernard [à ne pas confondre avec Jacques Bernard], Bruzen de La Martinière, Vauvenargues, Buffon, Diderot, d'Alembert, Helvétius, La Beaumelle, Boulanger, Morelly, Mably, d'Holbach, Rousseau avant le milieu des années 1750, et La Mettrie dans son matérialisme mais pas dans sa politique ou dans sa théorie morale ${ }^{46}$."

Malgré la confiance dont Israel fait preuve, ses assertions restent fort problématiques. En premier lieu, il est assez contestable de réunir sous une même étiquette tous ces philosophes, qui diffèrent aussi bien dans leurs productions que dans leurs idées. Deuxièmement, l'attribution d'un matérialisme athée à Fréret semble, quant à elle, au moins discutable. À cet égard, l'ouvrage qu'il faut examiner le plus attentivement est bien évidemment la Lettre de Thrasybule à Leucippe. Dans la préface à son édition critique du texte (1986), Sergio Landucci soutient avec conviction que Fréret en est le véritable auteur; cependant, il admet notamment que " nous n'en avons pas la preuve définitive, mais nous avons seulement une série d'indices et de témoignages ${ }^{47}$ ". De la même façon, Anthony McKenna affirme que la Lettre est authentique, en plaçant le travail de Fréret parmi d'autres " petits traités, dont l'ambition philosophique se limite à la critique radicale de certains dogmes de la religion chrétienne et de certains épisodes de l'histoire de son établissement ${ }^{48}$ ". Jean Deprun se range enfin du même côté que Landucci et McKenna. Sans se limiter à analyser soigneusement tous les témoignages en faveur de l'authenticité de la Lettre, il présente une comparaison minutieuse de la Lettre de Thrasybule avec d'autres travaux connus de Fréret. Il en conclut qu' " au terme de cette première série d'observations, l'hypothèse de l'authenticité semble bien recevoir plus qu'un commencement de preuve ${ }^{49}$ ". Néanmoins cette hypothèse ne manque pas susciter des critiques. On peut par exemple mentionner la position de Mario Sina, qui, dans son compte rendu de l'édition Landucci, s'oppose résolument à l'optimisme des partisans de l'authenticité :

" En particulier l'argument du parallèle entre les passages de la Lettre et ceux des ouvrages attribués avec certitude à Fréret n'est pas convaincant : cela peut juste prouver que l'auteur de la Lettre prend Fréret comme sa source, $[\ldots]$ et non pas qu'il s'identifie avec Fréret. [...] L'argument du double jeu, de la dissimulation, devrait être établi non seulement avec une référence vague et générique aux coutumes des "libertins" [...], mais avec une documentation précise et circonstanciée ${ }^{50}$."

46. Ibidem, p. 43.

47. FRÉRET, Nicolas, Lettre de Thrasybule à Leucippe, édition critique par LANDUCCI, Sergio, Firenze, Leo Olschki, 1986, p. 15.

48. McKenna, Antony, "Recherches sur la philosophie clandestine à l'Âge classique : bilan et perspectives ", dans McKennA, Antony, Mothu, Alain (éds.), La Philosophie clandestine à l'Âge classique, Paris, Universitas - Oxford, Voltaire Foundation, 1997, p. 9.

49. Deprun, Jean, " Une œuvre philosophique de la Régence. La Lettre de Thrasybule à Leucippe ", La Régence. Colloque d'Aix-en-Provence, les 24, 25 et 26 février 1968, Paris, Colin, 1970, p. 159.

50. SinA, Mario, "Recensione a N. Fréret, Lettre de Thrasybule à Leucippe, ed. critica, introduzione e commento a c. di S. Landucci ", Aevum, LXII, 3, 1988, p. 669. 
Pour la question qui est la nôtre - à savoir la formation intellectuelle de Maupertuis -, il importe cependant peu que Fréret soit le véritable auteur de cet ouvrage ou qu'il ne le soit pas.

Nous pouvons en effet rappeler grâce à lui que les premières années de Maupertuis à Paris coïncident à peu près avec la période de la Régence (1715-1723). Durant ces quelques années, parallèlement aux " œuvres consacrées à la défense de la foi ${ }^{51}$ ", il y a une diffusion considérable d'une "littérature matérialiste - et donc clandestine ${ }^{52}$ ". En ce sens, la Lettre de Thrasybule à Leucippe, toujours dans les mots de Deprun, est " l'un des textes les plus remarquables du "matérialisme de la Régence" 53 ", du fait qu'elle réunit les différents traits typiques des textes clandestins de cette période. Mais sans s'y limiter : les cercles intellectuels vraisemblablement fréquentés par Maupertuis à l'époque étaient un creuset de pareilles tendances radicales. De façon très significative, à côté de Fréret, un autre personnage mentionné constamment dans les récits ${ }^{54}$ est Nicolas Boindin. Ce dernier, lui aussi un habitué du café Procope ${ }^{55}$, professait un scepticisme dont La Beaumelle dans un cahier inédit souligne la radicalité : " Il dogmatisait le déisme et le pyrrhonisme; [...] Boindin a fait graver dans un cachet Descartes, Bayle et Fontenelle, ses maîtres de scepticisme : il les appelle sa Trinité ${ }^{56}$. "Boindin qui, comme Fréret, était membre de l'Académie des inscriptions, eut même un échange polémique avec Maupertuis au sujet des ses Réflexions philosophiques.

Ce qui importe ici, c'est que le jeune Maupertuis est sans doute entré en contact avec la pensée des libertins de son temps (les Fréret, Boindin, etc.), et que, selon toute vraisemblance, il a même lu quelques textes relevant de la tradition libertine. C'est là qu'il faut peut-être chercher l'origine du penchant pour la philosophie caractérisant son premier travail, mais bien plus évidemment ses travaux de maturité : en un certain sens, on pourrait même interpréter le goût maupertuisien pour la spéculation audacieuse, dont tous les commentateurs ont remarqué l'existence chez le Maupertuis de l'après-1740, comme l'élaboration et la ré-articulation de ces anciennes lectures.

51. Deprun, Jean, « Une œuvre philosophique de la Régence. La Lettre de Thrasybule à Leucippe... ", op. cit., p. 153.

52. Ibidem.

53. Ibid.

54. Cf. par exemple Duclos, Charles Pinot, Mémoires secrets sur le règne de Louis XIV, la Régence, et le règne de Louis XV, t. 1, Paris, Gay, 1864, p. 34-38.

55 . On a bien d'autres sources qui confirment la présence régulière de Boindin chez Procope. Voir par exemple ce qu'en dit Gustave Desnoiresterres dans La comédie satirique au XVIII ${ }^{e}$ siècle, Paris, Perrin, 1885, p. 35n : " Les tenants les plus illustres de Gradot étaient : Lamotte, Saurin, Nicole, Melon, Lafaye, Maupertuis, Duclos. Ceux du café Procope étaient, le plus généralement : Boindin, l'abbé Terrasson, Fréret, Piron, l'abbé Desfontaines. Mais l'on allait de l'un à l'autre, sans qu'à vrai dire, le personnel différât sensiblement. "

56. Cahier manuscrit de La Beaumelle cité dans LAURIOL, Claude, La Beaumelle. Un protestant cévenol entre Montesquieu et Voltaire, Genève, Droz, 1978, p. 176. 


\section{Les Modernes}

Revenons désormais au passage de la Vie de Maupertuis dans lequel La Beaumelle se réfère aux " citoyens " de la "république " en se réunissant chez Procope. Le biographe fait également mention d'Houdar de la Motte (le " dictateur perpétuel ") et de Terrasson. Ces derniers avaient notoirement pris part à la querelle des Anciens et des Modernes en se rangeant du côté des Modernes.

En 1714, Houdar de la Motte, alors qu'il ne connaissait pas le grec, publia une traduction en vers de l'Iliade en s'appuyant sur la version philologique en prose de Madame Dacier, parue quelques années auparavant ${ }^{57}$. En préface à sa traduction, La Motte ajoute aussi un Discours sur Homère, où il explique clairement sa méthode de travail : " J'ai suivi de l'Iliade ce qui m'a paru devoir être conservé, et j'ai pris la liberté de changer ce que j'y ai cru désagréable ${ }^{58}$ ". D'après La Motte, les défauts du poème homérique sont en effet nombreux : le récit est toujours " interrompu par de longs épisodes qui roulent sur les personnages indifférents, tandis qu'on perd de vue ceux qu'on voulait suivre ${ }^{59} "$; les dieux et les héros sont décrits d'une façon grossière, les combats sont monotones et ennuyeux ${ }^{60}$. Ces critiques acerbes de La Motte sont perçues chez les partisans des Anciens comme autant d'attaques de l'autorité la plus vénérable de l'Antiquité. La grande philologue Madame Dacier se charge donc de répondre aussitôt aux critiques de Houdar de La Motte, en publiant la même année un gros volume intitulé Des causes de la corruption du goût. Elle reproche à La Motte sa légèreté aussi bien que son incompétence (nous l'avons déjà mentionné, il ne connaissait pas le grec) : «Monsieur de La M. se contente d'ordinaire de la première appréhension des objets qu'il envisage, c'est pourquoi il se trompe si souvent ${ }^{61}$. " Et ceci n'est qu'un mince aperçu de la véhémence polémique dont fait preuve l'ouvrage de Madame Dacier.

Bien entendu, la dispute n'était pas destinée à s'épuiser rapidement, loin s'en faut. De plus en plus de lettrés commençaient à s'y atteler avec sérieux. C'est notamment le cas de Terrasson, qui est mentionné parmi les fréquentations de Maupertuis. Ses contributions à la Querelle présentent une pars construens d'un intérêt tout particulier. Pour se faire une idée de son état d'esprit, nous pouvons nous référer à ce qu'en dit Hippolyte Rigault, dans son Histoire de la Querelle (1856) :

57. L'lliade d'Homère traduite en français, avec des remarques. Par Madame Dacier, 3 vol., Paris, Rigaud, 1711.

58. HoudAR De La MotTe, Antoine, "Discours sur Homère ", dans LecoQ, Anne-Marie (éd.), La Querelle des Anciens et des Modernes. XVII -XVII ${ }^{e}$ siècles, précédé de Les abeilles et les araignées de Marc Fumaroli, postface de Jean-Robert Armogathe, Paris, Gallimard, 2001, p. 451.

59. Ibidem, p. 463.

60. Cf. aussi Rigault, Hippolyte, Histoire de la Querelle des Anciens et des Modernes, Paris, Hachette, 1856, p. 369-373.

61. LeFÈVRE-DACIER, Anne, " Des causes de la corruption du goût ", dans LECoQ, Anne-Marie (éd.), La Querelle des Anciens et des Modernes..., op. cit., p. 507. 
"Ses travaux en géométrie l'avaient conduit de bonne heure à l'Académie des sciences, mais ne l'avaient pas enrichi. Obligé pour vivre de faire l'éducation du fils de son cousin, [...] il avait, en dirigeant les études grecques de ce jeune homme, composé une longue dissertation en deux volumes sur l'lliade, et c'était de ce livre, prôné d'avance par les modernes, que La Motte prédisait la prochaine apparition à Mme Dacier : "il va venir un géomètre ennemi d'Homère" ${ }^{62}$."

Si on paraphrase Rigault, Terrasson était donc un géomètre qui avait été prêté à la littérature. Il avait l'intention d'affirmer, pour ainsi dire, la rationalité des mathématiques dans les belles-lettres, dans le but d'y introduire un principe de rationalité, à savoir la possibilité du " libre examen ${ }^{63}$ ". Ses détracteurs, par contre, lui reprochaient d'avoir sanctionné « la subordination de la littérature à la géométrie ${ }^{64}$ ". Plus généralement, Terrasson est habituellement présenté dans les récits comme un cartésien ${ }^{65}$, mais aussi - ce qui est encore plus intéressant - comme " un philosophe du Progrès ${ }^{66}$ ". S'il est vrai que sa conception téléologique de l'histoire se trouve pleinement développée seulement dans un ouvrage posthume, La Philosophie applicable à tous les objets de l'esprit et de la raison (1754), on trouve déjà l'essentiel de sa théorie du progrès dans les contributions données dans le cadre de la Querelle des Anciens et des Modernes : la Dissertation critique sur l' Iliade » d'Homère (1715), suivie par une Addition (1716). Dans ce texte, Terrasson identifie différents stades de développement de l'humanité dans l'histoire (sans pourtant en préciser exactement le nombre), qui mènent de " l'ignorance et la grossièreté ${ }^{67}$ " jusqu'à l'âge de la raison. En ce sens, il y a une correspondance presque parfaite entre histoire individuelle et universelle, entre l'enfant qui grandit lentement et l'humanité qui se perfectionne laborieusement. Cependant, comme Rigault le remarque, histoire individuelle et histoire universelle ne sont que partiellement superposables : le " philosophe optimiste [...] ne voit pas que si l'on compare le genre humain à l'individu, il faut pousser la comparaison jusqu'au bout. L'humanité [...] vieillit comme l'homme, en dépit de Terrasson, qui écarte vainement cette importune idée de la vieillesse ${ }^{68}$ ". En voulant justifier Terrasson, on peut ici rappeler que, dans l'ouvrage du 1754, il semble avoir conscience de la distinction entre particulier et général, quoique relativement à des points

62. Rigault, Hippolyte, Histoire de la Querelle..., op. cit., p. 417-418.

63. TERRASSON, Jean, " Dissertation critique sur l'Iliade d'Homère ", dans LECOQ, AnneMarie (éd.), La Querelle des Anciens et des Modernes..., op. cit., p. 601 : " Ma vue principale est de faire passer jusqu'aux belles-lettres cet esprit de philosophie qui depuis un siècle a fait faire tant de progrès aux sciences naturelles. "

64. Ibidem, p. 419.

65. D’AlEMBERT, Jean Le Rond, "Éloge de Jean Terrasson ", dans Id., Euvres complètes, t. 3, Paris, Belin et Bossange, 1821, p. 373 : "Sans être extrêmement zélé pour aucun système ni physique ni métaphysique, le cartésianisme était celui qu'il semblait avoir adopté." "

66. DelVAILLE, Jules, Essai sur l'histoire de l'idée de progrès jusqu'à la fin du XVIIe siècle, Genève, Slatkine Reprints, 2012 (réimpression de l'édition Paris, Alcan, 1910), p. 406.

67. TERRASSON, Jean, "Dissertation critique... ", art. cit., p. 605.

68. Rigault, Hyppolite, Histoire de la Querelle..., op. cit., p. 424. 
spécifiques - par exemple, l'exercice des facultés de l'imagination et du jugement ${ }^{69}$. Cependant, le point le plus important consiste dans la façon dont le philosophe caractérise le progrès. Contrairement à ce qu'on trouvera, par exemple, chez les positivistes du XIX ${ }^{\mathrm{e}}$ siècle, le progrès du genre humain n'est pas conçu chez Terrasson comme un progrès de nature essentiellement scientifique. Son raisonnement repose sur une conception de l'âme et de la vérité d'ascendance évidemment cartésienne : "Séparer la vue générale des progrès de l'esprit humain à l'égard des Sciences naturelles et à l'égard des Belles-Lettres, pourrait être un expédient convenable pour celui qui aurait deux âmes; mais il ne peut servir à rien à celui qui n'en a qu'une ${ }^{70}$. " Le domaine où le progrès se réalise étant si étendu, on peut, selon Terrasson, le désigner avec un mot seul : celui de philosophie. La philosophie, " n'étant pas elle-même qu'une lumière de l'esprit ${ }^{71}$ ", concerne la norme universelle de toutes les autres disciplines : voilà pourquoi les tournants décisifs dans l'histoire du progrès ne peuvent se limiter à une modification locale d'une théorie scientifique ou du seul goût artistique, mais ils doivent plutôt être retracés dans l'émergence de nouvelles conceptions du monde. C'est justement en ce sens que Terrasson peut se déclarer " cartésien ", même dans les années 1740 et 1750 . Il n'est pas question d'être partisan de Descartes contre Newton : il s'agit plutôt de reconnaître finalement que « Newton n'a point détruit Descartes [...]. Descartes est le premier auteur de ce qu'il y a de bon dans le Newtonisme; et cela dans les Points mêmes où le Newtonisme lui est contraire ${ }^{72}$ ". Et la raison de cela est bien manifeste : "Le Système de Descartes est un Système Philosophique; au lieu que celui de Newton n'est que Physique ou Géométrique ${ }^{73}$. "

Avant de quitter l'analyse de l'œuvre de Terrasson, il y a un dernier aspect de sa pensée qu'il faut bien prendre en compte, principalement parce qu'il nous semble en retrouver l'écho chez Maupertuis. D'après ce que nous avons dit jusqu'ici, on pourrait croire que l'attitude de Terrasson envers les Anciens est pétrie de mépris et d'hostilité. Tout au contraire :

69. TERRASSON, Jean, La philosophie applicable à tous les objets de l'esprit et de la raison. Ouvrage en réflexions détachées, Paris, Prault, 1754, p. 12 : "L'Homme pris en particulier, ne peut croître en un sens, qu'il ne décroisse en un autre : en acquérant la force du Jugement, il perd du feu de l'Imagination. Il n'est pas ainsi de l'Homme pris en général, ou du Public. parce qu'étant composé de tous les âges, il acquerra toujours au lieu de perdre. Rien ne peut lui nuire, que les événements qui ramèneraient la Barbarie. "

70. Ibidem, p. 20. De plus, Terrasson est persuadé que les anciens étaient bien plus avancés dans les sciences que dans les belles-lettres : "Quelques-uns de ceux qui font cette séparation conviennent que nous surpassons les Anciens dans les Sciences naturelles, en soutenant toujours que nous leur demeurons inférieurs dans la partie des Belles-Lettres. Or, j'ai prouvé que les Anciens connaissaient beaucoup mieux la Géométrie et l'Astronomie (quoiqu'il y fussent très-inferieurs à nous) qu'ils ne connaissaient les vrais principes de la Raison et de l'Humanité, unique force de véritable emploi des Belles-Lettres en Prose et en Poésie. " (Ibid.)

71. TERrasson, Jean, " Dissertation critique... ", art. cit., p. 615.

72. TERRASSON, Jean, La philosophie applicable à tous les objets de l'esprit et de la raison, op. cit., p. 9-10.

73. Ibidem, p. 9. 
Terrasson est profondément convaincu que sa théorie est la seule en mesure de sauvegarder une image positive de l'Antiquité. "Les partisans d'Homère " écrit-il, " ne doivent point s'opposer à notre gradation : elle est l'unique moyen de le sauver ${ }^{74}$ ". L'argument donné par Terrasson s'appuie sur la dialectique individu-histoire : si on ne tient pas compte de l'historicité des œuvres et des caractères, et qu'on ne juge de la supériorité des Anciens ou des Modernes qu'à partir de leurs travaux pris hors du temps (ou, ce qui est équivalent, en les considérant comme s'ils étaient tous contemporains), les Anciens en sortiront détruits. C'est justement ce qui se passe quand les partisans de l'Antiquité comparent directement Homère à Ronsard : la supériorité de ce dernier est manifeste. Mais si nous replaçons les auteurs dans leurs contextes historiques, en attribuant ainsi tous les défauts de l'Iliade à l'âge du genre humain où elle a été écrite, alors nous pourrons comprendre et finalement apprécier la véritable grandeur d'Homère.

"Le philosophe faisant une de ses principales occupations de l'étude de l'esprit humain sait se transporter aux siècles les plus éloignés, et en y reconnaissant l'ignorance et la grossièreté qui y étaient nécessairement attachées, il justifie la personne de toutes les fautes qu'on peut rejeter sur le temps. [...] Je leur [aux partisans des Anciens] accorderai même qu'Homère devait avoir beaucoup de génie pour faire ce qu'il a fait dans le temps où il a vécu ${ }^{75}$. "

En définitive, Terrasson ne tombe pas dans une exaltation naïve du génie moderne, mais aboutit à l'affirmation du caractère inévitable mais naturel, axiologiquement neutre, du progrès. Terrasson affirme que "les Progrès de l'Esprit humain dans le cours des Siècles, sont une suite d'une Loi naturelle exactement semblable à celle qui fait croître un Homme particulier en expérience et en sagesse depuis son enfance jusqu'à la vieillesse $^{76}$ ». Cela implique ultimement que " la supériorité des Modernes est "un effet naturel et nécessaire de la constitution de l'esprit humain" 77 ".

L'analyse que nous venons de proposer au sujet de la querelle des Anciens et des Modernes, ainsi qu'au sujet de l'apport original de Terrasson, n'est pas sans importance pour comprendre la présence chez Maupertuis des conjectures philosophico-historiques que nous avons évoquées. Ce lien paraît d'autant plus important que l'intérêt pour l'histoire de l'esprit ne se manifeste pas seulement dans le mémoire de 1724, mais c'est un leitmotiv qui accompagne la réflexion de Maupertuis jusqu'aux années de maturité ${ }^{78}$.

74. TERRASSON, Jean, « Dissertation critique... ", art. cit., p. 605.

75. Ibidem, p. 605-606.

76. TERRASSON, Jean, La philosophie applicable..., op. cit., p. 13.

77. Delvalle, Jules, Essai sur l'histoire de l'idée de progrès..., op. cit., p. 406.

78. Cf. par exemple la lettre Sur la génération des animaux de 1752, dans MAUPERTUIS, Pierre-Louis Moreau de, Euvres. Nouvelle édition augmentée et corrigée, 4 t., Lyon, JeanMarie Bruyset, 1768, II, p. 299-314. 


\section{La Société des arts}

La troisième et dernière source du jeune Maupertuis s'écarte sensiblement du milieu philosophique et littéraire commun soit aux esprits forts, soit aux Modernes de la querelle. Bien entendu, cela n'implique pas que les relations entre Maupertuis et cette troisième source se soient constituées hors des cafés et des salons. Par contre, c'est justement la nature des sujets de recherche, portant sur des thèmes plus proprement scientifiques, ainsi que le partenariat durable qui aurait dû s'instaurer entre Maupertuis et les membres de ce troisième groupement, qui impose de le distinguer très nettement des deux premiers. Nous pouvons débuter en citant quelques lignes du livre de Franco Venturi, Le origini dell'Enciclopedia (1963) :

" Déjà en 1726, on peut observer une tentative remarquable pour rapprocher la science de la technique [...]. Quelques jeunes savants, destinés à devenir par la suite célèbres dans différents domaines, s'étaient engagés dans une Société des Arts avec des inventeurs et techniciens pour comparer leurs expériences et collaborer à une œuvre qu'ils croyaient commune. Clairaut, mathématicien, Nollet, physicien et spécialiste de l'électricité, Rameau, théoricien de la musique ainsi que compositeur, y participaient avec Julien Le Roy, l'un des plus célèbres horlogers du dix-huitième siècle, et son fils Pierre. Et d'autres artistes : Sully, horloger anglais, l'architecte Chevotet, aussi bien que La Condamine et d'autres savants, ont travaillé pour faire vivre ce centre culturel d'un type nouveau ${ }^{79}$."

Il n'est pas difficile d'imaginer pourquoi il est tentant d'établir une quelque liaison entre Maupertuis et la Société des arts. D'abord, en fait, Clairaut fils et La Condamine avaient un rapport tout particulier avec le savant malouin. Comme Élisabeth Badinter le fait bien remarquer, Maupertuis allait devenir "le chef d'un petit clan très actif à l'Académie et hors d'elle. Au premier rang : deux amis intimes, également académiciens, le jeune génie Clairaut et La Condamine, au tempérament aventureux $^{80}$ ". Deuxièmement, déjà dans le mémoire Sur la forme des instruments de musique, on trouve plusieurs suggestions qui semblent indiquer, comme nous l'avons déjà mentionné, l'existence chez Maupertuis d'une référence directe aux activités de la Société des arts. Toutefois, on rencontre plusieurs difficultés lorsqu'on essaye de donner des arguments pour justifier cette relation. Pour discuter cette question en détail, il est nécessaire de donner un bref exposé de l'histoire de la Société des arts, qui permettra de fixer quelques éléments ${ }^{81}$.

Les sources s'accordent sur la date de démarrage de la Société, aussi bien que sur le but " technique " de l'entreprise. "Vers l'année 1726 ", écrit Joseph Bertrand en 1869, " Julien et Pierre Leroy et Henri Sully, célèbres

79. Venturi, Franco, Le origini dell'Enciclopedia, Torino, Einaudi, 1963, p. 14-15.

80. BADINTER, Élisabeth, Les passions intellectuelles, I. Désirs de gloire (1735-1751), Paris, Fayard, 1999, p. 57.

81. Une excellente source d'information pour tout ce qui concerne la Société des arts est représenté par le site internet [clairaut.com], réalisé par Olivier Courcelle. 
tous trois dans l'histoire de l'horlogerie, instituèrent des conférences réglées sur les moyens de perfectionner leur $\operatorname{art}^{82}$ ". Cependant, c'est seulement après la mort de Sully que la Société put officialiser son existence, grâce à la protection de l'abbé Bignon, obtenue en novembre 1728, et au patronage du comte de Clermont, dont le Mercure de France fit l'annonce début décembre ${ }^{83}$. Ce qui nous importe le plus reste néanmoins de comprendre la nature des travaux accomplis par cette société " pseudo-académique ", ou "proto-encyclopédique ". Dans son Éloge de M. le comte de Clermont, D'Alembert fournit une idée assez claire de la tâche que la Société des arts visait à accomplir, à savoir celle de "marier, pour ainsi dire, chaque art mécanique à la science dont cet art peut tirer des lumières, comme l'horlogerie à l'astronomie, la fabrique de lunettes à l'optique ${ }^{84}$ ». D'Alembert n'hésite pas à définir cet idéal comme un idéal « raisonnable ${ }^{85}$ ». Néanmoins, le jugement global exprimé par D'Alembert sur la Société est extrêmement sévère : " Le projet était grand, mais trop vaste, et fut d'ailleurs trop mal combiné par ceux que le Prince avait chargé de l'exécution ${ }^{86}$. " De fait, à côté de l'objectif défini comme " raisonnable ", il y en avait un autre qui, selon D'Alembert, était totalement dénué de sens. Il s'agissait en fait " [d'] accoler chacun de ces Arts à la partie des Belles-Lettres, qu'ils s'imaginaient y avoir le plus de rapport; par exemple, disaient-ils, le brodeur à l'historien, le teinturier au poète, et ainsi des autres ${ }^{87}$ ". Cette approche est également confirmée par des sources plus favorables à l'entreprise, comme l'Éloge de M. Clairaut de Fouchy, qui mentionne la contribution donnée par Clairaut "à l'établissement d'une Compagnie uniquement destinée à l'avancement des Arts; les Sciences y devaient aussi être admises, mais elles n'y jouaient pas le principal rôle, elle n'y paraissaient que pour aider les Artistes ou pour répondre à leurs questions ${ }^{88}$ ".

Quoique ce soit difficile à croire, l'émergence de la Société des arts n'est pas du tout un phénomène atypique dans son siècle. En 1963, Roger Hahn avait déjà catalogué un grand nombre de sociétés des arts parues tout au long du XVIII ${ }^{\mathrm{e}}$ siècle :

82. BERTRAND, Joseph, L'Académie des Sciences et les académiciens de 1666 à 1793, Hetzel, Paris, 1869, p. 95-96.

83. Mercure de France, 1728 (déc.), p. 2893 : « Cette société que l'on connaît aujourd'hui sous le nom de Société académique des beaux-arts, ayant eu l'honneur d'obtenir de S. A. S. M. le comte de Clermont la permission de s'assembler sous sa protection, alla le 7 décembre lui en faire ses très humbles remerciements. "

84. D'Alembert, Jean le Rond, "Éloge de M. le comte de Clermont ", dans Histoire des membres de l'Académie française morts depuis 1700 jusqu'en 1771, pour servir de suite aux éloges imprimés et lus dans les séances publiques de cette compagnie, t. 6, Paris, Moutard, 1787, p 347.

85. Ibidem.

86. Ibid.

87. Ibid., p. 347-348.

88. Fouchy, Jean-Paul Grandjean de, "Éloge de M. Clairaut ", Histoire de l'Académie Royale des Sciences, 1765, p. 149-150. 
"Jusqu'à présent, j'ai été en mesure de localiser presque cinquante institutions de ce genre dans toutes les régions du monde civilisé au dix-huitième siècle. Elles ponctuent la carte, de Saint-Pétersbourg à la côte brésilienne, de Florence à Édimbourg, en prenant racine surtout dans les centres urbains. Parmi ceux-ci, je ne citerai que les lieux principaux, avec la date probable de fondation : Édimbourg (1723), Paris (1726), Dublin (1731), Zurich (1743), Florence (1753), Londres (1753), Erfurt (1754), Rennes (1757), Berne (1759), New York (1764), Vergara (1765), Hambourg (1765), Saint-Pétersbourg (1766), Genève (1776), et Milan (1776) ${ }^{89}$. "

Mais si ces sociétés savantes avaient toutes une vocation purement technique, selon Hahn, celle de Paris était plutôt un homologue de l'Académie des sciences, en particulier en raison de la similarité de leurs règlements ${ }^{90}$ et de leurs structures internes. La Société des arts, de même que l'Académie, était ainsi divisée en classes et avait reçu des associés étrangers.

Pour en venir finalement à la trajectoire de la Société parisienne, on remarquera que, contrairement à la société dublinoise ${ }^{91}$, elle n'était pas destinée à une longue vie. Les membres de l'Académie des sciences officielle étaient en fait " inquiets des succès et de l'influence d'une compagnie nouvelle ${ }^{92}$ ", à tel point qu'ils s'engagèrent à faire tomber la Société; "Leur moyen fut très simple : l'Académie s'adjoignait successivement La Condamine, Clairaut, Fouchy, Nollet et Degua en leur imposant d'opter ${ }^{93}$. " La ruine définitive de cette " petite rivale et antichambre de l'Académie des sciences de Paris ${ }^{94}$ " ne devait pas tarder à se concrétiser : au début des années 1730, « la Société des arts, privée de ses membres les plus actifs, ne tarda pas à s'affaiblir et à tomber complètement, sans avoir produit aucune œuvre qui en perpétuât le souvenir ${ }^{95}$ ".

Comme nous le mentionnions plus haut, nous avons plusieurs raisons de supposer l'existence d'un lien entre les premiers travaux accomplis par Maupertuis au sein de l'Académie des sciences et les discussions de nature technique qui ont caractérisé la vie brève de la Société des arts. La rencontre de Maupertuis avec La Condamine, par exemple, remonte justement à cette époque-là, comme ils fréquentaient tous deux les mêmes

89. HaHn, Roger, " The Application of science to society : the societies of arts ", Studies on Voltaire and the Eighteenth Century, 25, 1963, p. 832.

90. Bibliothèque nationale de France, Ms. fr. 27662.

91. Cf. ClaRKE, Desmond J., A bibliography of the publications of the Royal Dublin Society from its foundation in the year 1731, together with a list of bibliographical material relative to the Society, Dublin, The Royal Dublin Society, 1953.

92. BerTRAnd, Joseph, L'Académie des Sciences..., op. cit., p. 97.

93. Ibidem.

94. Courcelle, Olivier, "Grandjean de Fouchy et la Société des arts à Stockholm ", Revue d'histoire des sciences, 61, 1, 2008, p. 203.

95. BERTRAnd, Joseph, L'Académie des Sciences..., op. cit., p. 97. Cependant, « la dernière trace administrative de la Société des arts est la lecture d'un mémoire de Le Maire, le 2 septembre 1736. La Société des arts est enterrée avec la parution, début 1737, de la réédition de l'œuvre maîtresse du fondateur Sully, augmentée de mémoires qui auraient dû trouver place dans les recueils de la Société, recueils qui ne verront jamais le jour " (COURCELLE, Olivier, [clairaut.com], section Société des arts). 
cercles savants : "lorsqu'il était à Paris à la fin des années 1720 ", écrit Neil Safier, " [La Condamine] fréquentait régulièrement le salon informel du café Procope $^{96}$ ». De même, Maupertuis connaît Clairaut fils lors de sa première apparition à l'Académie des sciences le 13 avril $1726^{97}$. En outre, comme nous l'avons déjà souligné, le contenu lui-même de l'ouvrage de Maupertuis suggère quelque affinité avec les finalités de la Société.

Il reste cependant une question d'envergure à envisager pour que la relation Maupertuis-Société des arts soit concevable : si (1) cette Société n'a commencé à opérer qu'à partir de 1726, et que (2) le premier mémoire maupertuisien a été rédigé en 1724, il s'ensuit que supposer que (2) dépend d'une certaine manière de (1) n'est rien d'autre qu'un anachronisme manifeste. Pire encore, un deuxième problème reste encore à résoudre. Dans la monographie de Beeson sur Maupertuis, nous lisons quelques mots qui semblent attester d'une opposition radicale de la part de Maupertuis à l’idéologie même de la Société :

"Quand La Condamine avait vingt-cinq ans et Clairaut treize, ils rejoignirent un groupe qui était concerné, non par les sciences abstraites, mais par les "arts", la technologie. Ils étaient plus familiers avec les vrais problèmes pratiques d'expérimentation que leurs adversaires dans l'Académie, y compris Maupertuis, qui avaient reçu une formation de rationalistes $^{98}$."

Aussi difficile que cela puisse être, nous tenterons de résoudre cette double difficulté. Quant à l'affirmation de Beeson, nous pouvons d'abord remarquer qu'elle n'est rien d'autre qu'une conjecture dénuée de tout fondement objectif. En effet, l'historien ne justifie pas son assertion par des documents. Ce qui, bien entendu, n'implique pas ipso facto la fausseté de ce qu'il dit; mais cela signifie que les affirmations de Beeson ne peuvent pas constituer un bon argument contre la position que nous essayons établir ici.

Concernant la question chronologique, par contre, il faut revenir au manuscrit Ms. fr. 22225, ff. 7-10, conservé à la Bibliothèque nationale de France, datant de l'année 1728. Le manuscrit, à l'origine attaché à la lettre de Liébaux à l'abbé Bignon du 8 novembre 1728, porte sur l'histoire de la Société des arts. Au tout début du manuscrit on lit :

"Cette société dont quelques-uns des membres ont été de celle des arts qui s'assemblait il y a quelques années aux galeries du Louvre avec l'approbation de feu M. le duc d'Orléans et sous la protection de Monsieur l'abbé Bignon a senti que la théorie seule n'allait guères plus loin qu'à satisfaire la curiosité, et que la pratique dénuée des lumières de la théorie n'était

96. SAFIER, Neil, Measuring the New World..., op. cit., p. 27.

97. Cf. le procès-verbal de $1726, \mathrm{f}^{\circ} 137 \mathrm{r}^{\circ}$ : "Monsieur Clairaut le fils âgé de 12 ans et 8 mois est entré et a lu un écrit de lui sur de nouvelles courbes dont il donne l'analyse par le calcul différentiel. " Encore une fois de grande utilité est le site [clairaut.com], section Clairaut et Maupertuis.

98. BEESON, David, Maupertuis: An Intellectual Biography..., op. cit., p. 99. 
ordinairement qu'une espèce d'habile routine que le bonheur du succès n'accompagnait pas toujours ${ }^{99}$."

Ces lignes font explicitement allusion à une activité de la Société des arts antérieure à 1726 durant la période où le duc d'Orléans était régent (1715-1723). Une confirmation supplémentaire vient du Mémoire pour servir à l'Histoire de l'Horlogerie de Julien Le Roy, membre de la Société des arts. Dans ce texte, publié en annexe à la Règle artificielle du temps (1737) de Sully, Le Roy se réfère encore d'une façon très claire à l'existence de la Société pendant la Régence :

"Vers ce temps-là, qui était celui de sa convalescence, il [Sully] proposa à Mr le Curé de Saint Sulpice de tracer une Méridienne dans le superbe Temple qu'il édifie à la gloire du Seigneur. Pendant qu'il travaillait à cet Ouvrage, pour lequel il avait beaucoup de goût, quelques-uns des membres de la Société des Arts, qui se tenait ci-devant au Louvre, sous la protection de M. le Régent, et à l'établissement de laquelle il avait beaucoup contribué, lui proposèrent de se joindre à eux pour en reprendre les Assemblées interrompues $^{100}$."

Roger Hahn lui-même, dans son ouvrage classique L'anatomie d'une institution scientifique (1971), confirme que les « origines [de la Société des arts] remontent à un groupe d'artistes non identifiés qui, avant la mort du Régent en 1723, se rencontraient périodiquement aux Galeries du Louvre ${ }^{101}$ ".

Nous pouvons finalement l'affirmer avec assurance : la possibilité que les discussions autour de la naissante Société des arts aient influencé Maupertuis dans l'élaboration de son premier travail académique ne peut pas être exclue $a$ priori.

L'analyse que nous venons de proposer avait pour but d'indiquer quelques difficultés ressortant de la lecture du mémoire Sur la forme des instruments de musique, pour ensuite essayer d'en donner une explication plausible. Une telle étude, dans nos intentions, constitue un premier pas vers la redécouverte de la pensée de Maupertuis, ainsi qu'une première tentative de le placer dans le réseau plus vaste de la République des Lettres.

À notre avis, la tâche qui reste à accomplir consiste dans l'analyse d'autres moments importants dans la biographie du savant malouin, - à

99. BnF, Ms. fr. $22225, f^{\circ} 7$ (nos italiques).

100. LE Roy, Julien, "Mémoire pour servir à l'Histoire de l'Horlogerie, depuis 1715 jusqu'en 1729 ", dans Sully, Henri, Règle artificielle du temps, Traité de la division naturelle et artificielle du Temps, des Horloges et des Montres de différentes constructions, de la manière de le connaître et de les régler avec justesse, Paris, Dupuis, 1737, p. 407-408 (nos italiques).

101. HAHN, Roger, L'anatomie d'une institution scientifique. L'Académie des Sciences de Paris, 1666-1803, Amsterdam, Éditions des archives contemporaines, 1993, p. 152. Olivier Courcelle reprend exactement les mêmes mots : « Du temps de la régence (1715-1723), une première mouture de la Société des arts s'assemble dans les galeries du Louvre avec l'approbation du duc d'Orléans et sous la protection de l'abbé Bignon " (COURCELLE, Olivier, [clairaut.com], section Société des arts). 
commencer par la rencontre avec l'œuvre de Newton -, sans jamais perdre de vue les contextes socioculturels au sein desquels ces travaux ont été rédigés. C'est seulement en mettant dans cette perspective l'œuvre de Maupertuis qu'on pourra vraiment comprendre sa portée et juger de sa cohérence, aussi bien que passer d'un simple récit biographique à l'examen d'un processus historique plus complexe et riche.

\section{RÉSUMÉ}

Le premier mémoire académique de Pierre-Louis Moreau de Maupertuis (1698-1759), intitulé Sur la forme des instruments de musique (1724), a été souvent négligé par les historiens. Cela est d'autant plus étonnant si l'on considère les difficultés évidentes qu'il pose d'un point de vue à la fois historique et théorique. Pour essayer de résoudre ces problèmes d'interprétation, dans la présente contribution nous avons approfondi les étapes principales de la première formation intellectuelle de Maupertuis, aussi bien que les sources possibles de quelques-unes de ses doctrines. Nous sommes bien conscients que cette opération ne nous livrera rien d'absolument certain. Dans le pire des cas, toutefois, nous aurons esquissé un cadre historico-philosophique à l'intérieur duquel le savant malouin a débuté sa carrière : une préhistoire de l'activité savante de Maupertuis qui, à ce jour, reste encore largement inexplorée.

\section{ABSTRACT}

Historians have constantly neglected Pierre-Louis Moreau de Maupertuis's (1698-1759) first academic work, On the Shape of the Musical Instruments (1724). This is all the more surprising if we consider the issues it raises from both an historical and a theoretical point of view. In order to solve such issues, in this paper we will give a thorough analysis of the main stages of Maupertuis's intellectual apprenticeship, and of the possible sources of some of his doctrines as well. We are well aware that such an operation will yield nothing absolutely certain. In the worst case, however, we will have sketched an historical-philosophical framework within which the Breton savant took his first steps: a pre-history of Maupertuis's scientific activity, which remains still largely unknown. 